\title{
On the use of Dynamic Programming in eco-driving cycle computation for electric vehicles
}

\author{
D. Maamria $^{a}$, K. Gillet ${ }^{a}$, G. Colin ${ }^{a}$, Y. Chamaillard ${ }^{a}$ and C. Nouillant ${ }^{b}$
}

\begin{abstract}
This paper considers the problem of eco-driving for electric cars. This problem is formulated as an Optimal Control Problem (OCP) aiming at minimizing the vehicle's energy consumption over fixed time and distance horizons. The impact of battery parameter variations and auxiliary power demands on the optimal vehicle velocity computation are studied from a model complexity viewpoint. Simulation results are presented and discussed to illustrate the suggested simplifications.
\end{abstract}

\section{INTRODUCTION}

The expected depletion of fossil fuel sources, climate change due to pollution and an increase in overall energy demands are major challenges for the automotive industry. More generally, energy efficiency is increasingly becoming a major concern. For this purpose, highly efficient powertrain and lightweight automobiles are being developed. Furthermore, eco-driving is now considered as a major solution to reduce the energy consumption linked to transportation system.

In this context, several studies investigating the problem of the vehicle speed trajectory optimization have been reported [1]-[4]. In these studies, an Optimal Control Problem (OCP) to determine the velocity trajectory that minimizes the energy consumption under final time and distance constraints was formulated and solved. In particular, the eco-driving problem for electric vehicles was addressed in [1]-[3]. Usually, two state variables are considered in the OCP: the position and the speed of the vehicle.

In [1], the eco-driving problem was studied and solved for an electric car powered by a DC-type motor. The model used was based on an analytical expression of the electric power demanded by the electric machine. In [2], the same problem using similar modeling assumptions as [1] was addressed for an electric car powered by a permanent-magnet synchronous machine by taking into account physical limitations on the control actions. The advantage of the proposed solution is its relatively low computational time compared to Dynamic Programming (DP). Later, in [3], a more representative (realistic) model of the electric machine was used while neglecting the dependance of the internal battery resistance and the open circuit voltage on the battery State Of Charge (SOC) [5] as commonly assumed in the literature dealing with energy management system design for hybrid electric vehicles [6]-[9].

${ }^{a}$ D. Maamria, K. Gillet, G. Colin and Y. Chamaillard are with Laboratoire PRISME, Université d'Orléans 45072 Orléans, France djamaleddine.maamria@gmail.com

${ }^{b} \mathrm{C}$. Nouillant is with PSA Peugeot Citroën, Direction Recherche Innovation \& Technologies Avancées (DRIA), France
In this paper, a similar eco-driving problem to [3] is considered. The dependance of battery parameters on the SOC is taken into account in the optimization. This increases the number of state variables from 2 (vehicle position and speed) to 3 (SOC, vehicle position and speed). The objective is to select the right level of modeling to optimize the accuracy/complexity trade-off. A main motivation is that the number of state variables greatly impacts the used numerical methods. Considering additional state variables increases the level of complexity and the computational burden. This observation holds for DP, and for methods using Pontryagin Minimum Principle (PMP) or direct formulations (e.g. collocation methods) [10], [11]. A special focus, in this paper, is on the quantification of the gain in energy consumption by including the SOC dynamics in the optimization.

The paper is organized as follows. In Section 2, the vehicle model is described. The calculation of eco-driving cycles and the questions addressed in this paper are detailed in Section 3. Section 4 discusses numerical and simulation results. In light of the results, some conclusions on the trade-off between the complexity of the models used to calculate ecodriving cycles and the optimality of the associated solutions are drawn.

\section{VEHICLE MODELING}

\section{A. Motion equations}

The vehicle is modeled on the longitudinal axis. The motion of the vehicle is the result of the forces that are applied on its body. According to Newton's law of motion, the vehicle speed $v$ satisfies the differential equation:

$$
\left(m+m_{r o t}\right) \cdot \frac{d v(t)}{d t}=F_{t}(t)-F_{r}(t),
$$

where $F_{t}$ is the traction force to be provided by the electric machine, $F_{r}$ is the sum of resistance forces and $m$ is the total vehicle mass. The term $m_{r o t}$ is an equivalent mass of the rotating parts. It accounts for the overall inertia of the wheels $\left(n_{\text {tire }} \cdot j_{\text {tire }}\right)$ and for that of the electric machine $\left(j_{\text {rot }}\right)$ :

$$
m_{\text {rot }}=\frac{n_{\text {tire }} \cdot j_{\text {tire }}+j_{\text {rot }}}{r_{\text {tire }}^{2}},
$$

where $r_{\text {tire }}$ is the wheel radius. The force $F_{r}$ comprises the rolling resistance force, the aerodynamic drag force and a force due to the road grade. Its expression is given by:

$$
F_{r}(t)=c_{0}+c_{1} \cdot v(t)+c_{2} \cdot v(t)^{2},
$$

where $c_{i}, i=\{0,1,2\}$ are the coefficients of the road load equation (this expression of $F_{r}$ was employed in [6], [12]). 
This model considers only the forces in the longitudinal direction. Variations of friction parameters during curves, wind forces, and other disturbances are neglected.

\section{B. Transmission model}

The driver's torque demand and the vehicle speed are directly calculated from the wheel speed profile, elevation profiles and the transmission ratio. The resulting torque value $T_{w h}$ can be positive (traction) or negative (braking). The electric machine torque $T_{e}$ is related to the torque requested at the wheel $T_{w h}$ by:

$$
T_{w h}(t)=r_{\text {tire }} \cdot F_{t}(t)=\eta_{t} \cdot R_{t} \cdot T_{e}(t),
$$

where $\eta_{t}$ is the transmission efficiency and $R_{t}$ is the constant motor-to-wheel transmission ratio. Similarly, the rotational speed $\omega_{e}$ of the electric machine is related to the vehicle speed $v$ by:

$$
\omega_{e}(t)=R_{t} \cdot \frac{v(t)}{r_{\text {tire }}} .
$$

\section{Electric machine model}

The electric machine is modeled by a quasi-static map describing either the electric power or its efficiency. The electric power $P_{m}$ consumed (in traction mode) or supplied to the battery (in recuperation mode) is of the form:

$$
P_{m}=P_{m}\left(T_{e}, \omega_{e}\right)
$$

where $P_{m}$ is the electric power map of the electric machine.

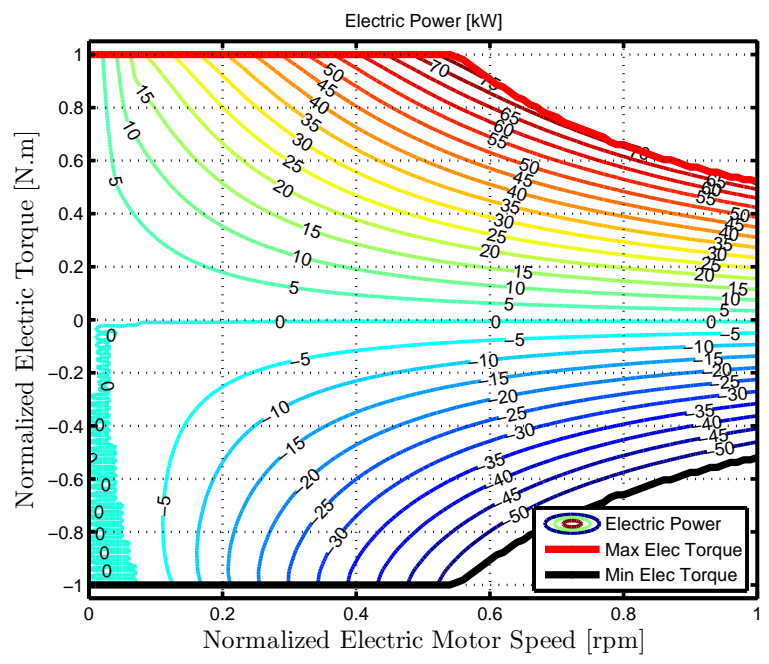

Fig. 1. Electric Machine Power $[\mathrm{kW}]$

This map includes the losses in the electric machine and the power electronic devices. The electric machine torque is limited by speed-dependent upper and lower bounds of the form (bold red and black lines in Figure 1):

$$
T_{\text {emin }}\left(\omega_{e}\right) \leq T_{e} \leq T_{\text {emax }}\left(\omega_{e}\right)
$$

\section{Auxiliary power demand model}

A constant or a piecewise constant power demanded by auxiliaries $P_{a u x}$ (radio/tape player, lights, air conditioning and heating systems) is considered. This power is provided by the battery.

\section{E. Battery model}

The battery is usually represented by an equivalent circuit model comprising a voltage source $U_{o c v}$ in series with an electric resistance $R_{b}$, both of which vary with $\xi$, the battery state of charge (SOC) [6], [13]. The expression of the battery current $I_{b}$ is given by [6]:

$$
I_{b}=\frac{1}{2 R_{b}(\xi)}\left(U_{o c v}(\xi)-\sqrt{U_{o c v}^{2}(\xi)-4 R_{b}(\xi) \cdot P_{b}}\right),
$$

where $P_{b}$ is the power requested from the battery given by:

$$
P_{b}=P_{m}+P_{a u x} .
$$

The dynamics of $\xi$ is given by:

$$
\frac{d \xi(t)}{d t}=-\frac{I_{b}(t)}{Q_{0}}
$$

where $Q_{0}$ is the nominal battery capacity. In order to simplify the notation, the dynamics of $\xi$ considering a given initial condition $\xi_{0}$ is written as:

$$
\frac{d \xi(t)}{d t}=g\left(v(t), \xi(t), T_{e}(t)\right), \xi(0)=\xi_{0} .
$$

The inner (electrochemical) battery power is defined by:

$$
P_{e c h}\left(v, \xi, T_{e}\right)=I_{b}\left(v, \xi, T_{e}\right) \cdot U_{o c v}(\xi) .
$$

The model parameters are summarized in Table I.

TABLE I

VEHICLE MODEL PARAMETERS

\begin{tabular}{|l|l|c|c|}
\hline & Description & Value & Unit \\
\hline$m$ & Vehicle mass & 1300 & $\mathrm{~kg}$ \\
$r_{\text {tire }}$ & Wheel radius & 0.34 & $\mathrm{~m}$ \\
$n_{\text {tire }}$ & Wheel number & 4 & - \\
$j_{\text {tire }}$ & Wheel inertia & 4.28 & $\mathrm{~kg} \cdot \mathrm{m}^{2}$ \\
$c_{0}$ & Constant coefficient of road load & 120.86 & $\mathrm{~N}$ \\
$c_{1}$ & Linear coefficient of road load & 0.3 & $\mathrm{~N} /(\mathrm{m} / \mathrm{s})$ \\
$c_{2}$ & Quadratic coefficient of road load & 0.0377 & $\mathrm{~N} /(\mathrm{m} / \mathrm{s})^{2}$ \\
$\eta_{t}$ & Transmission efficiency & 0.925 & - \\
$R_{t}$ & Motor-to-wheel transmission ratio & 4.7647 & - \\
$Q_{0}$ & Battery nominal capacity & 28800 & $\mathrm{C}$ \\
$R_{b}$ & Internal battery resistance & $1.2-1.6$ & $\mathrm{~m} \Omega$ \\
$U_{\text {ocv }}$ & Open circuit voltage & $3.45-4.10$ & $\mathrm{~V}$ \\
\hline
\end{tabular}

\section{ECO-DRIVING}

An eco-driving methodology consists in finding the optimal way to reduce the overall energy consumption [2], [14]. For a given road, the objective is to find the best speed profile minimizing the vehicle power consumption knowing that the vehicle starts from a point $A$ at rest and must reach a destination point $B$ in a duration $t_{f}$, with a zero velocity. This kind of question can be formulated as an OCP [4], [14].

\section{A. Problem under consideration}

The objective in this study is to investigate the impact of the battery parameters SOC dependance and the auxiliaries power demand on the optimal speed trajectory. The battery parameters SOC dependance is studied here from a model complexity viewpoint to find a trade-off between the computation time (induced by the model complexity) and 
the optimality of the solution (energy consumption). Two questions are addressed:

1) What is the maximum benefit of considering the dependance of battery parameters in the optimal speed trajectory calculation?

2) What is the impact of the auxiliaries power demand $\mathrm{P}_{a u x}$ on the optimal speed trajectory?

\section{B. OCP formulation}

The cost function (4) to be minimized is the electrochemical battery energy in traction over a fixed time window of duration $t_{f}$ :

$$
J(u)=\int_{0}^{t_{f}} P_{e c h}(v(t), \xi(t), u(t)) d t,
$$

where the control variable $u$ is the electric machine torque:

$$
u(t)=T_{e}(t)
$$

This optimization is carried out under the following differential equations:

$$
\begin{aligned}
\frac{d v(t)}{d t} & =f(v(t), u(t)), v(0)=0, \\
\frac{d x(t)}{d t} & =v(t), x(0)=0, \\
\frac{d \xi(t)}{d t} & =g(v(t), \xi(t), u(t)), \xi(0)=\xi_{0},
\end{aligned}
$$

where $x$ is the position of the vehicle and the function $f$ is calculated by combining $(1,2,3)$ :

$f(v, u)=\frac{1}{m+m_{\text {rot }}}\left(-c_{0}-c_{1} \cdot v-c_{2} \cdot v^{2}+\frac{\eta_{t}}{r_{\text {tire }}} \cdot R_{t} \cdot u\right)$.

Since the speed and the electric machine torque are limited and the final position and speed are set, the optimization must be performed under the following state and input constraints:

$$
\begin{aligned}
v(t) & \in\left[0, v_{\max }(x(t))\right] \\
u(t) & \in\left[T_{e \min }\left(\omega_{e}(t)\right), T_{\text {emax }}\left(\omega_{e}(t)\right)\right], \\
x\left(t_{f}\right) & =D \\
v\left(t_{f}\right) & =0
\end{aligned}
$$

where $D$ is the total traveled distance. The speed limits are given as a function of the vehicle position and not of time [15].

On the other hand, the final value of $\xi$ is free as the traction of the vehicle is ensured only by the electric energy (no additional energy source is available on board as for hybrid electric cars).

To summarize, the following OCP can be defined:

$$
(O C P): \min _{u} J(u)
$$

under the dynamics $(5,6,7)$, state and input constraints $(8$, 9) and the final constraints $(10,11)$.

\section{Simplified $O C P$}

The dynamics of $\xi$ is taken into account in the (OCP) described in (12) because of the internal battery resistance $R_{b}$ and the open circuit voltage $U_{o c v}$ dependance on $\xi$. To reduce the calculation time and the model complexity, mean constant values of $R_{b}$ and $U_{o c v}$ can be considered in the model used to calculate the optimal solution. In this case, the cost function (4) becomes of the form:

$$
J_{s}(u)=\int_{0}^{t_{f}} P_{e c h}(v(t), \bar{\xi}, u(t)) d t,
$$

where $\bar{\xi}$ is a fixed value used to calculate mean values of $R_{b}$ and $U_{o c v}$. As the cost function $J_{s}$ and the dynamics of $v$ and $x$ are independent of $\xi$, the number of the state variables is thus reduced from $3(v, x, \xi)$ to $2(v, x)$. The following simplified OCP can be defined:

$$
\left(O C P_{s}\right): \min _{u} J_{s}(u)
$$

under the dynamics $(5,6)$, state and input constraints $(8,9)$ and the final constraints $(10,11)$.

\section{Numerical solving method}

The OCPs defined in (12) and (13) can be solved by numerous methods. The solution considered here is based on Dynamic Programming (DP) [16]. Considering the cost function $J$ to be minimized:

$$
J=\int_{0}^{t_{f}} L(X(t), u(t), t) d t
$$

from a mathematical viewpoint, Bellman's principle can be formulated as follows: let $t \in\left[0, t_{f}\left[\right.\right.$ and $\mathbf{X}(t) \in \mathbb{R}^{n}$ be given, then for all real $r \in\left[t, t_{f}\right]$, the cost-to-go function $V$ satisfies:

$V(X(t), t)=\min _{u \in \mathcal{U}}\left\{\int_{t}^{r} L(X(\tau), u(\tau), \tau) d \tau+V(X(r), r)\right\}$

This equation is solved recursively and backward. In order to reduce the calculation time, the method suggested in [3], [4], [17] is used, where an additional tunable term $\beta \cdot t_{f}$ is added to the cost function as a terminal cost:

$$
\begin{aligned}
& \bar{J}(u)=\int_{0}^{t_{f}} P_{e c h}(v(t), \xi(t), u(t)) d t+\beta \cdot t_{f}, \\
& \bar{J}_{s}(u)=\int_{0}^{t_{f}} P_{e c h}(v(t), \bar{\xi}, u(t)) d t+\beta \cdot t_{f} .
\end{aligned}
$$

The constant tunable parameter $\beta$ penalizes the final time to obtain almost the same time duration as the initial driving cycle. Thus, two new OCPs can be defined as follows:

$$
\begin{array}{cc}
(\overline{O C P}): & \min _{u} \bar{J}(u), \\
\left(\overline{O C P}_{s}\right): & \min _{u} \bar{J}_{s}(u)
\end{array}
$$

To calculate the right value of the tunable parameter $\beta$, a root-finding method can be used to drive the final time error to zero as done in [15], [18]. It was shown in [3], [4] that the solutions of the problems $(\overline{O C P})$ and $\left(\overline{O C P}_{s}\right)$ converge to the solutions of $(O C P)$ and $\left(O C P_{s}\right)$ respectively when the obtained final time is almost the same as the initial driving cycle. 


\section{NuMERICAL RESULTS}

To compute an eco-driving cycle, the following constraints [14] extracted from an initial driving cycle have to be considered:

- the same final distance $x\left(t_{f}\right)$, the same number of stops and almost the same duration $t_{f}$ as the initial driving cycle.

- the vehicle speed limits depending on the position of the vehicle $(x)$.

To specify the speed limits, the following legal speed limits $v_{\text {lim }}$ were applied:

$$
v_{l i m}=[30,50,70,90,110,130,150](\mathrm{km} / \mathrm{h}) .
$$

The choice of this vector is not restrictive. Other speed limits can be used. The process of identifying the appropriate speed limit can be described in two steps:

1) For each time $t$, find the maximum value of $j$ for which $v_{\text {lim }}(j)<v(x(t))$ and $v_{\text {lim }}(j) \geq v(x(t))$.

2) $v_{\max }(x(t))=v_{\text {lim }}(j)$.

Two normalized driving cycles are considered: the EUDC cycle with a duration of $360 \mathrm{~s}$ and traveled distance of $6.9 \mathrm{~km}$ and the Worldwide harmonized Light vehicles Test Cycle (WLTC) with a duration of $1574 \mathrm{~s}$ and traveled distance of $22.7 \mathrm{~km}$.

The two OCPs defined in (14) and (15) are solved using DP with the following grid parameters: $\delta v=0.01 \mathrm{~m} / \mathrm{s}$ for the vehicle speed and of $\delta x=20 \mathrm{~m}$ for the distance. For the control input $u$, a $\delta u=1 \mathrm{~N} . \mathrm{m}$ in the case of the EUDC Cycle and $\delta u=2 \mathrm{~N} . \mathrm{m}$ in the case of the WLTC cycle are used. The notation $\delta \xi$ refers to the step of $\xi$ (SOC) used to solve the OCP (14). The initial value of the SOC is $\xi(0)=90 \%$.

To make a fair comparison between the solutions of problems (14) and (15), the control trajectory in each case is applied to a (forward) simulation model considering the variation of $R_{b}$ and $U_{o c v}$ as functions of $\xi$.

\section{A. Impact of SOC dependent battery parameters}

The internal resistance $R_{b}$ and the open circuit voltage $U_{\text {ocv }}$ depend on the SOC value. The maximum variation of $R_{b}$ is about $12 \%$ while the variation of the $U_{o c v}$ is $14 \%$ when the SOC changes between $90 \%$ and $20 \%$. The impact of this variation on the optimal speed calculation is studied below for two driving cycles.

1) EUDC cycle: The speed trajectories obtained from the DP are shown in Figure 2 versus distance and in Figure 3 versus time. The electric energy consumption in [\%] and the time $\alpha$ needed to run the DP are given in Table II.

\section{TABLE II}

TIME $\alpha$ NEEDED TO RUN THE DP AND $\xi(0)-\xi\left(t_{f}\right)$ FOR EUDC CYCLE.

\begin{tabular}{|l|c|c|}
\hline & $\xi(0)-\xi\left(t_{f}\right)[\%]$ & $\alpha[\mathrm{s}]$ \\
\hline EUDC: Initial Cycle & 18.74 & - \\
\hline Eco-EUDC: Simplified model & 16.74 & 16 \\
\hline Eco-EUDC: $\delta \xi=1 \%$ & 16.73 & 764 \\
\hline Eco-EUDC: $\delta \xi=0.5 \%$ & 16.73 & 1537 \\
\hline
\end{tabular}

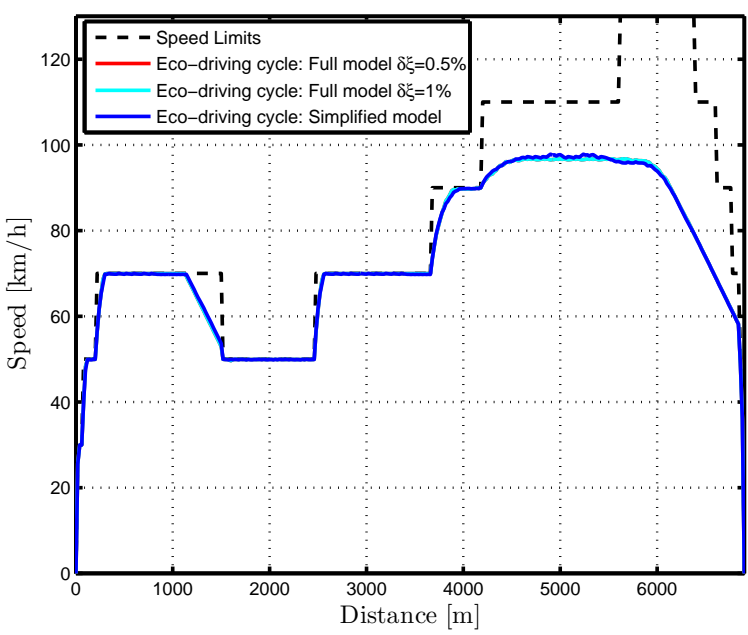

Fig. 2. Vehicle speed $[\mathrm{km} / \mathrm{h}]$ vs distance $[\mathrm{m}]$ for EUDC cycle.

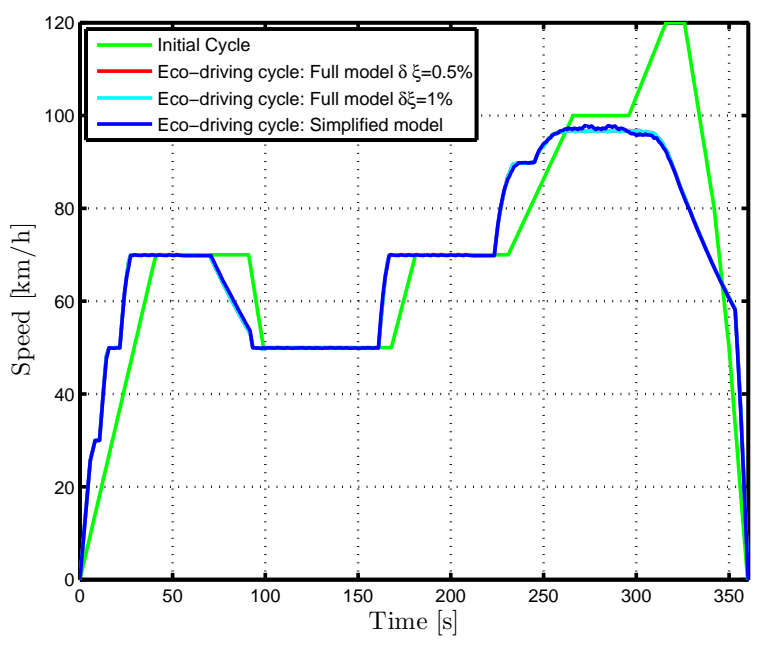

Fig. 3. Vehicle speed $[\mathrm{km} / \mathrm{h}]$ vs Time $[\mathrm{s}]$ EUDC cycle.

Figures 2 and 3 show that the eco-speed trajectories satisfy the speed limits and that they are close. This can be confirmed from Table II where the difference in the final SOC between the cycles Eco-EUDC for $\delta \xi=0.5 \%$ and Eco$E U D C$ with the simplified model is negligible $(0.01 \%$ on the energy consumption). On the other hand, the difference in the time needed to calculate these two cycles is significant (a ratio of 96).

2) WLTC cycle: For the previous case, the variation range of the SOC is not high. In order to ensure that the SOC sweeps a larger range, the WLTC cycle is used. The speed profiles obtained are shown in Figure 4 versus distance and in Figure 5 versus time. The electric energy consumption in [\%] and the time $\alpha$ needed to run the DP are given in Table III. we highlight that the stops are indicated in distance and not in time as for the speed limits.

Figures 4 and 5 show that the eco-speed trajectories satisfy the speed limits. From Table III, the difference in the final SOC between the cycles Eco-WLTC for $\delta \xi=1 \%$ and 


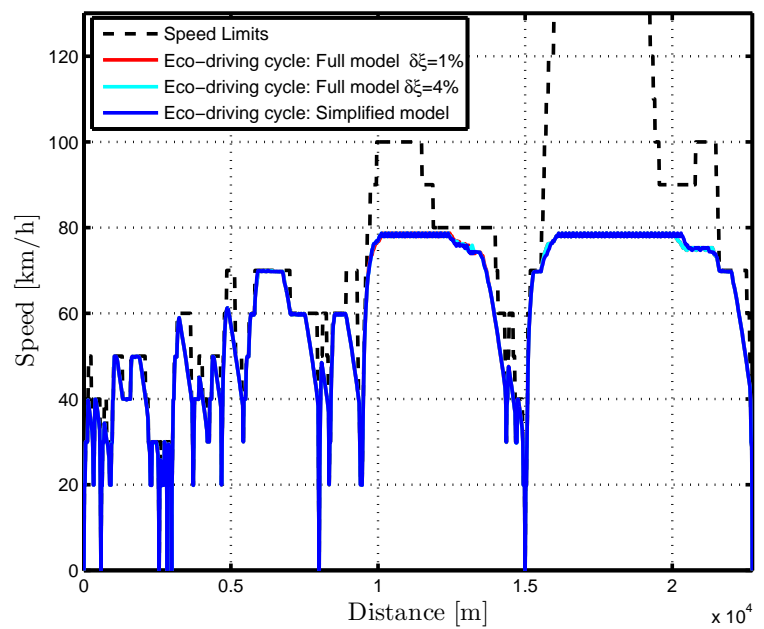

Fig. 4. Vehicle speed $[\mathrm{km} / \mathrm{h}]$ vs distance $[\mathrm{m}]$ for WLTC.

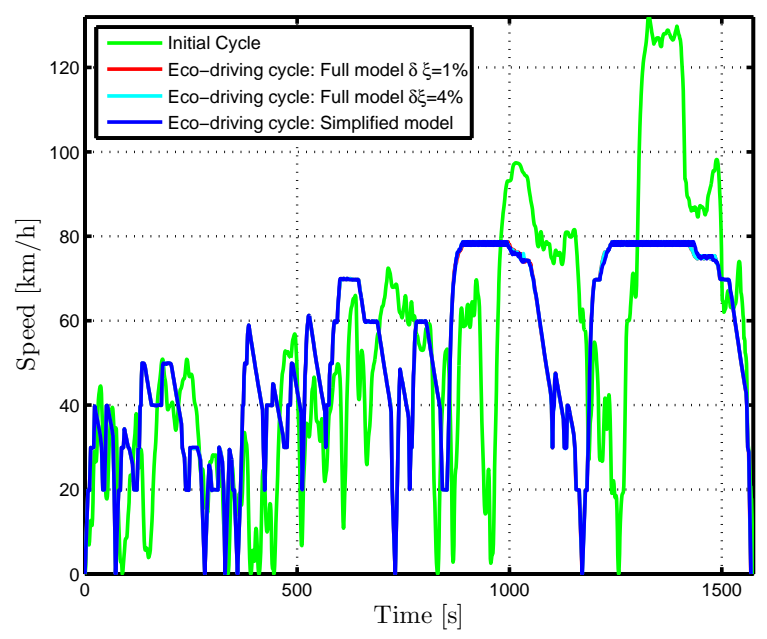

Fig. 5. Vehicle speed $[\mathrm{km} / \mathrm{h}]$ vs Time [s] for WLTC.

Eco-WLTC with the simplified model is $0.01 \%$ (which is considered as negligible). On the other hand, the time needed to run the DP increases by a ratio of 116 when considering $\xi$ in the OCP.

3) Conclusion: Because of the negligible sub-optimality induced and the reduction in the calculation time, the numerical results presented above suggest that it is sufficient to solve the OCP defined in (15) to calculate the optimal speed trajectory and to take the battery parameters dependance on the SOC into account only in the (forward) simulation model. Note that similar analysis have been done for other normalized driving cycles: NEDC, Urban Artemis, Artemis Rural and Artemis highway cycles.

\section{B. Impact of the auxiliaries power demand}

From the conclusion of the previous section, the OCP defined in (15) is considered in what follows. This optimization problem is solved in the case of the EUDC cycle (with a duration of 360s) for various values of $\beta$.
TABLE III

TIME $\alpha$ NEEDED TO RUN THE DP AND $\xi(0)-\xi\left(t_{f}\right)$ FOR WLTC.

\begin{tabular}{|l|c|c|}
\hline & $\xi(0)-\xi\left(t_{f}\right)[\%]$ & $\alpha[\mathrm{s}]$ \\
\hline WLTC: Initial Cycle & 80.23 & - \\
\hline Eco-WLTC: Simplified model & 62.82 & 30 \\
\hline Eco-WLTC: $\delta \xi=4 \%$ & 62.82 & 800 \\
\hline Eco-WLTC: $\delta \xi=1 \%$ & 62.81 & 3500 \\
\hline
\end{tabular}

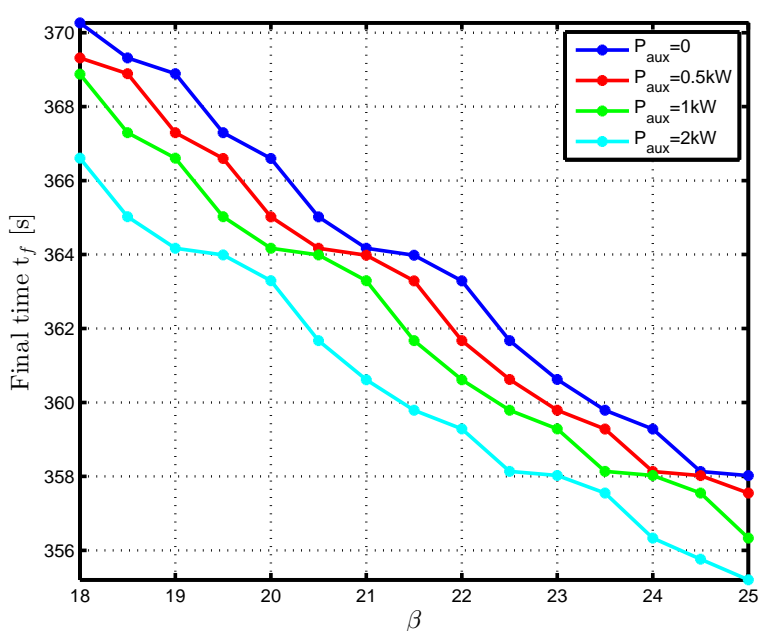

Fig. 6. Relation between $\beta$ and the final time $t_{f}$ [s].

1) Constant auxiliaries power demand: Various constant auxiliaries power demand $P_{a u x}$ are considered in the OCP (15). The final time $t_{f}$ and the electric energy consumption are given in Figures 6 and 7. As one can see from Figures 6 and 7, the auxiliaries power demand $P_{\text {aux }}$ shifts the final time $t_{f}$ for the same value of $\beta$ : the final time decreases when $P_{a u x}$ increases.

The objective here is to study the impact of $P_{a u x}$ on the speed trajectories for the same final time $t_{f}$. The speed trajectories having the same $t_{f}$ for various values of $P_{a u x}$ are given in Figure 8. These results indicate that the optimal speed profiles are the same for the values of $P_{a u x}$ considered. The difference is only on the final SOC as illustrated in Figure 7 for a final time $t_{f}$ around 360s.

2) Variable auxiliaries power demand: A variable auxiliaries power demand in $[\mathrm{kW}]$ defined by:

$$
P_{a u x}=\left\{\begin{array}{l}
1, \text { if } x(t) \leq \frac{D}{3} \\
0.5, \text { if } \frac{D}{3}<x(t) \leq \frac{2 D}{3}, \\
0, \text { if } x(t)>\frac{2 D}{3},
\end{array}\right.
$$

is considered. The optimal speed trajectory obtained for this variable $P_{\text {aux }}$ is compared to the optimal speed calculated for $P_{a u x}=0$ in Figure 9 (the two solutions must have the same final time). The optimal speed profiles are the same for the two cases.

3) Conclusion: The numerical results suggest that it is sufficient to set $P_{\text {aux }}$ to zero in the model used to calculate 


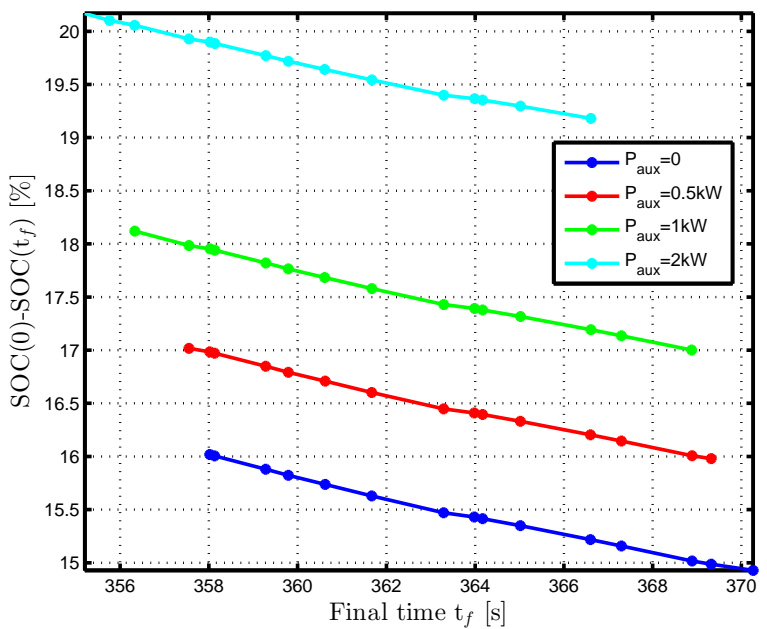

Fig. 7. Relation between $t_{f}[\mathrm{~s}]$ and energy consumption $\xi(0)-\xi\left(t_{f}\right)[\%]$.

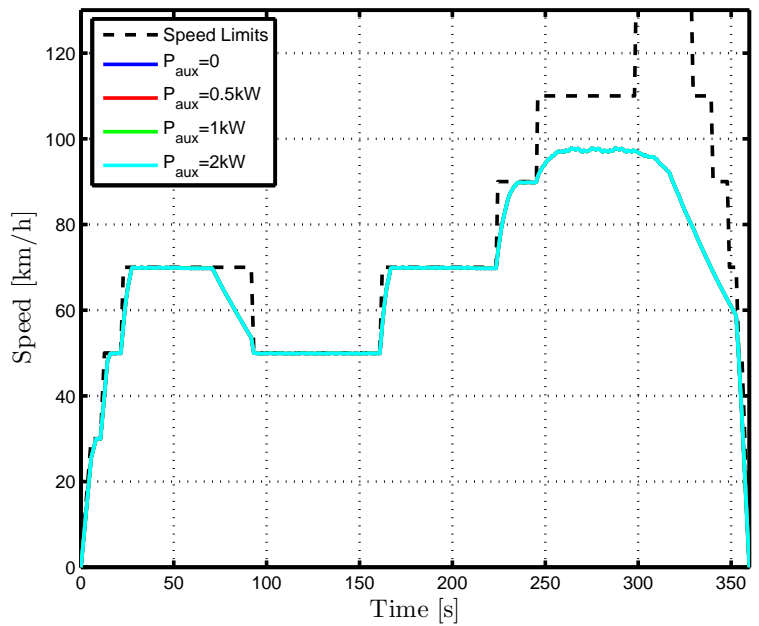

Fig. 8. Speed trajectories for various constant values of $\mathrm{P}_{\text {aux }}$.

optimal speed trajectories and to take it into account only in the forward simulation model.

\section{CONCLUSION}

The eco-driving problem for electric vehicles has been addressed. This problem is formulated as an OCP aiming at minimizing the electric energy consumption. The studies conducted suggest that is not necessary to take into account the battery parameters dependence on the SOC and the auxiliaries power demand on the optimization problem. The impact of these simplifications on the optimal speed trajectory is negligible while ensuring a reasonable time to run dynamic programming.

\section{REFERENCES}

[1] N. Petit and A. Sciarretta, "Optimal drive of electric vehicles using an inversion-based trajectory generation approach," 18th IFAC World Congress, vol. 18, pp. 14519-14526, 2011.

[2] W. Dib, A. Chasse, P. Moulin, A. Sciarretta, and G. Corde, "Optimal energy management for an electric vehicle in eco-driving applications," Control Engineering Practice, vol. 29, pp. 299-307, 2014.

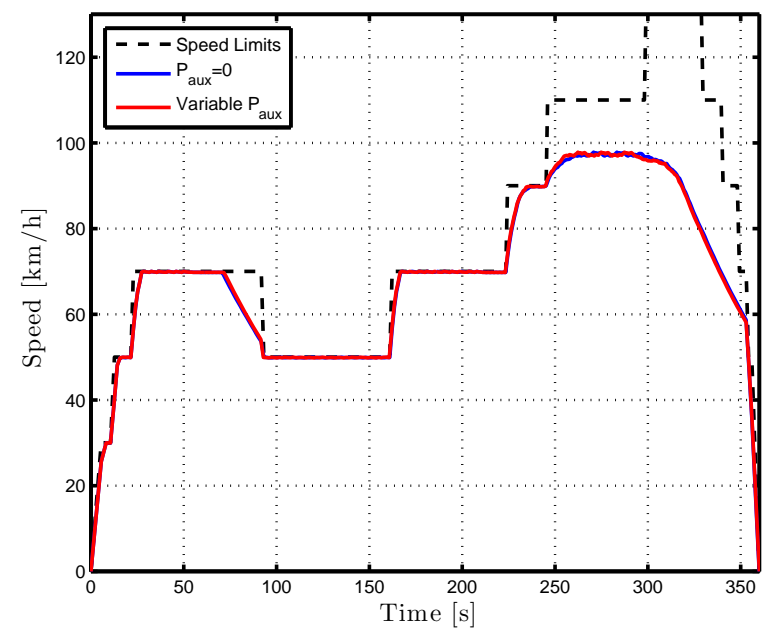

Fig. 9. Speed trajectories for a variable $\mathrm{P}_{a u x}$ defined in (16).

[3] F. Mensing, "Optimal energy utilization in conventional, electric and hybrid vehicles and its application to eco-driving," Ph.D. dissertation, INSA Lyon, 2013.

[4] V. Monastyrsky and I. Golownykh, "Rapid computation of optimal control for vehicles," Transportation Research Part B, vol. 27, pp. 219-227, 1993.

[5] M. Miyatake, M. Kuriyama, and Y. Takeda, "Theoretical study on ecodriving technique for an electric vehicle considering traffic signals,' in Proc. 9th IEEE Int. Conf. Power Electronics Drive Systems, pp. 733-738, 2011.

[6] L. Guzzella and A. Sciarretta, Vehicle propulsion systems. Springer, 2013.

[7] A. Sciarretta, M. Back, and L. Guzzella, "Optimal control of parallel hybrid electric vehicles," IEEE Transactions on Control Systems Technology, vol. 12, no. 3, pp. 352-363, 2004.

[8] L. Serrao, S. Onori, A. Sciarretta, Y. Guezennec, and G. Rizzoni, "Optimal energy management of hybrid electric vehicles including battery aging," American Control Conference, 2011.

[9] T. van Keulen, B. de Jager, D. Foster, and M. Steinbuch, "Velocity trajectory optimization in hybrid electric trucks," in Proc. American Control Conference, pp. 5074-5079, 2010.

[10] M. Athans and P.-L. Falb, Optimal control: an introduction to the theory and its applications. Dover, 2006.

[11] C.-R. Hargraves and S.-W. Paris, "Direct trajectory optimization using nonlinear programming and collocation," Journal of Guidance, Control and Dynamics, vol. 10, pp. 338-342, 1987.

[12] S-B. Ebbesen, "Optimal sizing and control of hybrid electric vehicles," Ph.D. dissertation, ETH Zurich, 2012.

[13] F. Badin, Hybrid Vehicles. Editions TECHNIP, 2013.

[14] F. Mensing, E. Bideaux, R. Trigui, J. Ribet, and B. Jeanneret, "Ecodriving: an economic or ecologic driving style?" Transportation Research Part C: Emerging Technologies, vol. 38, pp. 110-121, 2014.

[15] F. Mensing, R. Trigui, and E. Bideaux, "Vehicle trajectory optimization for application in eco-driving," IEEE Vehicle Power and Propulsion Conference, pp. 1-6, 2011.

[16] D. Bertsekas, Dynamic programming and optimal control. Athena Scientific, 2012.

[17] H. Bouvier, G. Colin, and Y. Chamaillard, "Determination and comparison of optimal eco-driving cycles for hybrid electric vehicles," European Control Conference, pp. 142-147, 2015.

[18] A. Sciarretta, G. D. Nunzio, and L. L. Ojeda, "Optimal ecodriving control: Energy-efficient driving of road vehicles as an optimal control problem," IEEE Control Systems Magazine, 2015. 